University of Wollongong

Research Online

Faculty of Social Sciences - Papers (Archive) Faculty of Arts, Social Sciences \& Humanities

$1-1-2016$

\title{
Ability of measures of adiposity in identifying adverse levels of inflammatory and metabolic markers in adolescents
}

J Oliveira-Santos

University of Porto

Rute Santos

University of Wollongong, rutes@uow.edu.au

Carla Moreira

University of Porto

Sandra Abreu

University of Porto

Luis Lopes

University of Porto

See next page for additional authors

Follow this and additional works at: https://ro.uow.edu.au/sspapers

Part of the Education Commons, and the Social and Behavioral Sciences Commons

Research Online is the open access institutional repository for the University of Wollongong. For further information contact the UOW Library: research-pubs@uow.edu.au 


\title{
Ability of measures of adiposity in identifying adverse levels of inflammatory and metabolic markers in adolescents
}

\begin{abstract}
Background: Overweight and obesity have been associated with a pro-inflammatory state. We aimed to assess the ability of different measures of overall and abdominal adiposity for identifying adverse levels of inflammatory and metabolic markers in adolescents. Methods: This is a cross-sectional analysis with 529 Portuguese adolescents (267 girls), mean age $14.3 \pm 1.7$ years. Weight, height, sitting height, waist circumference (WC), and body fat percentage (BF\%) were measured; and $\mathrm{BMI}$, waist-to-height ratio (WHtR), and waist-to-sitting-height ratio (WsHtR) were calculated. We measured C-reactive protein (CRP), fibrinogen, erythrocyte sedimentation rate (ESR), complements C3 and C4, leptin, and adiponectin levels. Receiver operating characteristic (ROC) curves were used to estimate the ability of the adiposity measures to discriminate between low/high values of biomarkers. Results: The highest areas under the ROC curves were presented by BF\% for fibrinogen and complement $\mathrm{C} 3$ in both sexes and for ESR, complement C4, and adiponectin only in girls; by BMI for CRP in girls and for leptin in both sexes; by WHtR for leptin in both sexes and for CRP, fibrinogen, and adiponectin only in girls; by waist circumference for CRP, fibrinogen, and complement $\mathrm{C} 3$ only in boys and for complement $\mathrm{C} 4$ in girls; and by WsHtR for complement $\mathrm{C} 3$ in girls; $\mathrm{p}<0.05$ for all. Conclusions: The measures that more often presented discriminatory power were, for overall adiposity, BF\% in both sexes, and for abdominal adiposity, WHtR in girls and WC in boys. However, small differences in discriminatory capabilities don't allow us to clearly defend the adoption of a single measure above all others.
\end{abstract}

\section{Keywords}

adolescents, markers, metabolic, inflammatory, levels, ability, adverse, measures, identifying, adiposity

\section{Disciplines}

Education | Social and Behavioral Sciences

\section{Publication Details}

Oliveira-Santos, J., Santos, R., Moreira, C., Abreu, S., Lopes, L., Agostinis, C. \& Mota, J. (2016). Ability of measures of adiposity in identifying adverse levels of inflammatory and metabolic markers in adolescents. Childhood Obesity, 12 (2), 135-143.

\section{Authors}

J Oliveira-Santos, Rute Santos, Carla Moreira, Sandra Abreu, Luis Lopes, C Agostinis-Sobrinho, and Jorge Mota 


\section{Ability of different measures of adiposity in identifying adverse levels \\ of inflammatory and metabolic markers in adolescents}

José Oliveira-Santos ${ }^{*}, \mathrm{MSc}^{1}$, Rute Santos, $\mathrm{PhD}^{1,2}$, Carla Moreira, $\mathrm{PhD}^{1}$, Sandra Abreu, $\mathrm{PhD}^{1}$, Luís Lopes, $\mathrm{PhD}^{1}$, César Agostinis, $\mathrm{MSc}^{1}$ \& Jorge Mota, $\mathrm{PhD}^{1}$

${ }^{1}$ Research Centre in Physical Activity, Health and Leisure (CIAFEL) - Faculty of Sport of the University of Porto, Portugal.

Rua Dr. Plácido Costa, 91. 4200-450 Porto, Portugal.

Phone number: 00351220425200

Fax number: 00351225500689

${ }^{2}$ Early Start Research Institute, Faculty of Social Sciences. University of Wollongong, Australia.

Wollongong NSW 2522, Australia.

\section{* Corresponding Author:}

José Oliveira-Santos

Email: jomios@gmail.com

Rua Dr. Plácido Costa, 91. 4200-450 Porto

Phone number: 00351220425200

Fax number: 00351225500689 


\section{ABSTRACT}

BACKGROUND: Overweight and obesity have been associated with a pro-inflammatory state. We aimed to assess the ability of different measures of overall and abdominal adiposity in identifying adverse levels of inflammatory and metabolic markers in adolescents.

METHODS: This is a cross-sectional analysis with 529 Portuguese adolescents (267 girls), mean age $14.3 \pm 1.7$ years. Weight, height, sitting height, waist circumference and body fat percentage (BF\%) were measured, and body mass index (BMI), waist-to-height ratio (WHtR) and waist-to-sitting-height ratio $(\mathrm{WsHtR})$ were calculated. We measured $\mathrm{C}$-reactive protein (CRP), fibrinogen, erythrocyte sedimentation rate (ESR), complements $\mathrm{C} 3$ and $\mathrm{C} 4$, leptin and adiponectin levels. Receiver operating characteristic (ROC) curves were used to estimate the ability of the adiposity measures to discriminate between low/high values of biomarkers.

RESULTS: The highest areas under the ROC curves were presented by: BF\% for fibrinogen and complement $\mathrm{C} 3$, in both sexes, and for ESR, complement C4 and adiponectin, only in girls; BMI for CRP in girls, and for leptin in both sexes; WHtR for leptin in both sexes, and for CRP, fibrinogen and adiponectin, only in girls; waist circumference for CRP, fibrinogen and complement $\mathrm{C} 3$, only in boys, and for complement C4, in girls; WsHtR for complement $\mathrm{C} 3$, in girls. $p<0.05$ for all.

CONCLUSIONS: The measures that more often presented discriminatory power were, for overall adiposity, BF\% in both sexes, and for abdominal adiposity, WHtR in girls and WC in boys. However, small differences in discriminatory capabilities don't allow us to clearly defend the adoption of a single measure above all others.

KEYWORDS: Adolescents, obesity, anthropometry, biomarkers, ROC curves. 


\section{INTRODUCTION}

Overweight and obesity have been associated with a condition of chronic low-grade systemic inflammation even in children ${ }^{1,2}$ and adolescents ${ }^{3,4}$, with evidence of tracking into adulthood ${ }^{5,6}$ contributing to an increased risk of cardiovascular disorders and diabetes ${ }^{7}$ over time.

Several anthropometrical measures, indices and techniques have been used in epidemiological studies to assess obesity in youngsters, as alternatives to more accurate but hardly feasible laboratory assessment, because of the constraints in time, sample mobilization and high costs. ${ }^{8}$ Although body mass index (BMI) is probably the most widely used index to define weight status across populations, ${ }^{9,10}$ other techniques also provide information on overall adiposity, such as bioelectric impedance and skinfold measurements, with estimates of body fat percentage (BF\%).

In addition, other anthropometric measures have focused on abdominal obesity, a known independent risk factor for insulin resistance and cardiovascular diseases in children and adolescents. ${ }^{11,12}$ Low-grade systemic inflammation in youth has been shown to be associated with high waist circumference (WC), ${ }^{13}$ waist-to-hip ratio (WHR) ${ }^{14}$ and waist-toheight ratio $(\mathrm{WHtR}) .{ }^{15}$ Moreover, individuals with a greater percentage of visceral fat appear to have higher levels of some circulating cytokines and acute-phase reactants than individuals with a higher percentage of subcutaneous fat, ${ }^{16}$ suggesting that visceral fat could play a more active role in the development of systemic inflammation.

Therefore, the search for one anthropometric measure (or more) that may adequately provide sensitivity and specificity to reflect not only adiposity, but also an adverse inflammatory condition, seems pertinent.

In light of the heterogeneity and lack of previous studies, we aimed to examine and compare different measures of overall obesity such as BMI and BF\%, and central obesity like WC, WHtR and waist-to-sitting-height ratio (WsHtR), with respect to their ability to detect increased levels of inflammatory and metabolic markers in a sample of Portuguese adolescents, trying to verify which measure presents the greatest predictive power for each biomarker. 


\section{SUBJECTS, MATERIALS AND METHODS}

\section{Study design and sampling}

We used baseline data from the Longitudinal Analysis of Biomarkers and Environmental Determinants of Physical Activity Study (LabMed Physical Activity Study), a school-based prospective cohort study carried out in five schools from the north of Portugal, which aimed to evaluate the independent and combined associations of dietary intake and fitness levels on blood pressure levels of adolescents. The power calculation for that study was based on the exposure of combined healthy diet/physical activity pattern with a prevalence of $14 \% .{ }^{17}$ A sample of 754 participants would provide $80 \%$ power to detect $15 \%$ difference between exposed and unexposed at 5\% significance, but taking into account an expected dropout rate of about $20 \%$ at each time-point, the minimum sample size was increased to 1086. Baseline data was collected in the fall of 2011, for all pupils that agreed to participate in the study $(n=1229)$. From this initial total sample of apparently healthy adolescents (12-18 years old), 534 agreed to undergo blood collection. Five of them were later excluded from the analysis due to high sensitivity C-reactive protein (CRP) values $>10 \mathrm{mg} / \mathrm{L}$, which may be indicative of acute inflammation or illness, ${ }^{18}$ leaving 529 adolescents (267 girls, 262 boys, mean age $14.3 \pm 1.7$ years) as the final sample for the present study. This number fulfills the condition of a Receiver Operating Characteristic (ROC) sample size calculation (providing $80 \%$ power at $5 \%$ significance, for a minimum expected AUC of 0.6 and null hypothesis value of 0.5 ) requiring at least 514 subjects for the present study.

This study was conducted in accordance with the Helsinki Declaration for Human Studies of 1975 , as revised in 2008, and approved by the Portuguese Data Protection Authority (\#1112434/2011) and the Portuguese Ministry of Science and Education (0246200001/2011). All participants were informed of the study's goals and all procedures were carried out with the adequate understanding of the subjects, and written informed consent was obtained from participating adolescents and their parents/tutors.

\section{Anthropometric measurements}

Anthropometric measurements were performed according to standardized procedures, ${ }^{19}$ with all participants lightly dressed with a t-shirt, shorts and barefoot. 
Body weight was measured to the nearest $0.1 \mathrm{~kg}$, using a portable electronic weight scale (Tanita InnerScan BC532, Tokyo, Japan). Body height was measured to the nearest 0.1 $\mathrm{cm}$, with the adolescent standing upright against a portable stadiometer (Seca213, Hamburg, Germany). BMI was calculated as weight divided to height squared $\left(\mathrm{kg} / \mathrm{m}^{2}\right)$ and the participants were classified as underweight, normal weight, overweight or obese using the age and sex-specific cut-off values proposed by the International Obesity Task Force. ${ }^{9,20}$

Waist circumference was taken in a standing position, midway between the lower rib margin and the anterior superior iliac spine, at the end of a normal expiration, to the nearest $0.1 \mathrm{~cm}$ with a non-elastic tape measure. WHtR was calculated as the WC divided by height, both measured in centimetres.

Sitting height was measured with the participant seated on a table, with back and buttocks positioned against a stadiometer and with the head positioned in a Frankfort horizontal plane. The participant's knees were directed straight ahead, with the arms and hands resting at the sides. Sitting height was measured from the tabletop to the vertex and recorded to the nearest $0.1 \mathrm{~cm}$. WsHtR was also calculated as the WC divided by sitting height.

BF\% was measured by bioelectrical impedance ${ }^{21}$ (Tanita InnerScan BC532) according to manufacturer's instructions, in the morning after an overnight fast for at least 10 hours, and 2 hours after last water intake and urination. Participants were asked not to perform any physical exercise before the measurements that morning. The device used is suitable for measuring body fat percentage in an age range from 7 to 99 years old according the instruction manual, and has already been used for the same purpose in other studies with adolescents. $^{22}$

\section{Pubertal stage assessment}

Pubertal stage (breast and pubic hair development in girls and genital and pubic hair development in boys, with stage 1 being pre-pubertal and 5 being adult) was self-assessed by the participants according to the classification by $T a n n e r,{ }^{23}$ in a private place, and then communicated to a researcher of the same sex in a closed envelope. 


\section{Biochemical assessment}

After an overnight fast (>10 hours), blood samples were collected between 8:0010:00 a.m. by venipuncture from the antecubital vein. The samples were stored in sterile blood collection tubes in refrigerated conditions $\left(4^{\circ}\right.$ to $\left.8^{\circ} \mathrm{C}\right)$ for no longer than 4 hours during the morning of collection, and then delivered to an analytical laboratory for testing according to standardized procedures, as follows: (i) high sensitivity CRP, latex enhanced immunoturbidimetric assay (Siemens ADVIA 1800, Erlangen, Germany); (ii) fibrinogen, Clauss assay (Siemens BCS XP System, Erlangen, Germany); (iii) adiponectin and leptin, ELISA (Plate Reader); (iv) complement factor C3 (C3) and complement factor C4 (C4), PEG enhanced immunoturbidimetric assay (Siemens ADVIA 1800, Erlangen, Germany); (v) erythrocyte sedimentation rate (ESR), Westergren method (Starrsed, RR Mechanotronics, Netherlands). CRP, C3, C4, adiponectin and leptin were determined in serum, fibrinogen was determined in plasma and ESR was determined in whole blood. The existing literature shows that all the biomarkers analyzed in this study have been increasingly explored in studies involving children or adolescents ${ }^{24-27}$, suggesting that they are a valid choice for this age group.

\section{Statistical analyses}

Two-sided Student's $t$-Test was used for comparisons between groups.

For each biomarker, a Z-score was computed by age and sex, and increased levels were considered when the individual had $\geq 1$ SD of the Z-score, except for adiponectin, where decreased levels were considered when the individual had $\leq-1$ SD of the Z-Score. The setting of $\geq 1 S D$ (and $\leq-1 S D$ in the case of adiponectin) allowed us to identify the adolescents of our sample with the highest values for each biomarker (or lowest, in the case of adiponectin), showing two statistically different groups in relation to the biomarkers mean values (data not shown), and consequently create two categories for the ROC curves analysis.

ROC curves analyzed the ability of different measures of adiposity to discriminate between low/high values of inflammatory and metabolic markers, providing the best trade-off between sensitivity and specificity of each adiposity measure and respective cut-off value. 
The area under the curve (AUC), ranging between 0 and 1 (a worthless and a perfect test, respectively), represents the ability of the test to correctly classify the participants with high or low inflammatory markers. ROC curve analysis showed which measures of adiposity performed well in identifying increased levels of inflammatory and metabolic markers. When the AUC $>0.5$ and $p<0.05$ was considered that the ROC performed well. Cut-off points were chosen based in the highest Youden index.

Data were analysed using the Statistical Package for Social Sciences version 22.0 (SPSS, IBM Corp., NY, USA), and MedCalc statistical software version 15 (MedCalc software, Mariakerke, Belgium) was used for all the ROC curves analyses, including the sample size calculation for this study. A $p$-value $<0.05$ denoted statistical significance.

\section{RESULTS}

Participants' characteristics are shown in Table 1. Boys were heavier, taller, and had higher WC than girls, while girls presented higher values of $B F \%$ than boys ( $<<0.05$ for all). In relation to the biomarkers, only CRP values were higher in boys, whereas fibrinogen, adiponectin, erythrocyte sedimentation rate and leptin were higher in girls ( $p<0.05$ for all).

\section{INSERT TABLE 1}

Tables 2 and 3 presents ROC analysis for the associations of measures of overall (BMI and BF\%) and abdominal obesity (WC, WHtR and WsHtR) with biomarkers for girls and boys, respectively. Values in bold represent the ROC curves indicating the adiposity measures that presented the highest AUC for each biomarker.

$\mathrm{BF} \%$ was the measure of overall adiposity that more often presented the best discriminatory power for both sexes. Higher levels of fibrinogen were suggested by BF\% cutoff values $>27.3 \%$ for girls and $>18.3 \%$ for boys, and of C3 by BF\% cut-off values $>30.7 \%$ in girls and $>18.2 \%$ in boys. BF\% also showed discriminatory power for high levels of ESR and C4 and lower levels of adiponectin, but only for girls. BMI presented the best trade-off between sensitivity and specificity for leptin in girls and boys, and for CRP in girls.

Regarding abdominal adiposity, WC presented the best discriminatory power for higher levels of CRP, fibrinogen, and C3 in boys, and for C4 in girls. WHtR showed the best trade-off between sensitivity and specificity for detecting in girls increased levels of CRP, 
fibrinogen and adiponectin, and higher levels of leptin in both girls and boys. WsHtR only demonstrated the best discriminatory power for $\mathrm{C} 3$ in girls.

\section{INSERT TABLES 2 AND 3}

\section{DISCUSSION}

The main findings of this study showed that for measures of overall adiposity, the highest AUC were presented by BF\% for fibrinogen and complement $\mathrm{C} 3$, in both sexes, and for ESR, complement C4 and adiponectin, only in girls; and by BMI for CRP in girls, and for leptin in both sexes. Regarding measures of abdominal adiposity, the highest AUC were presented by WHtR for leptin in both sexes, and for CRP, fibrinogen and adiponectin, only in girls; by WC for CRP, fibrinogen and complement C3, for boys, and for complement C4, in girls; and by WsHtR for complement $\mathrm{C} 3$, in girls.

Some studies suggested associations between measures of overall ${ }^{2,3}$ and abdominal ${ }^{28,29}$ obesity and a series of inflammatory and metabolic markers. However, to the best of our knowledge, there is currently no agreement about which anthropometric measures predict best adverse levels of inflammatory markers, and how they differ by sex.

Our results suggest that measures of overall adiposity tended to more often present higher AUC in girls, whereas in boys higher AUC were more frequent in measures of abdominal obesity.

Regardless of sex, BF\% showed marginally higher differences in the AUC for most of the inflammatory markers compared with BMI. BF\% coincided for boys and girls as the measure of overall adiposity that best predicts increased levels of fibrinogen and C3, with slightly higher pooled AUC observed in girls compared to boys, suggesting that discrimination is more precise, on average, in girls. For CRP, ESR, C4 and adiponectin, BF\% only showed discriminatory power for girls.

BMI was the best measure of overall adiposity for both sexes predicting higher levels of leptin, but showing this time an inverse trend, with a higher AUC for boys. In agreement with other studies, ${ }^{30,31}$ we found that leptin and adiponectin levels were significantly lower in boys than in girls. It is hypothesized that the increase in testosterone level during male puberty could play an important role in the drop of the expression of these adipokines, ${ }^{32}$ and 
boys from our study were mainly in pubertal stages 4 and 5 .

$\mathrm{BMI}$ also presented a slightly higher AUC than BF\% predicting CRP, but this time in girls. Analysis of the data from the National Health and Nutrition Examination Survey (NHANES) $^{3,33,34}$ suggests that BMI is a good predictor of elevated CRP in children, and has been widely used to predict body composition and health-related risks. ${ }^{9,10}$ Indeed, in a recent systematic review and meta-analysis ${ }^{35}$ the random-effects summary correlation between BMI and CRP in children and adolescents was strong $(r=0.37 ; 95 \% \mathrm{Cl}=0.31-0.43)$.

Nevertheless, it is important to emphasize that BMI is a measure of excess weight relative to height, and does not provide information on the distribution of body fat nor differentiates between fat and lean mass ${ }^{36}$ In this study we could observe that for a similar BMI value for age, girls and boys are different in relation to body composition, indicated by a significant higher BF\% in girls. In a 7.5 -year longitudinal study, Wen et al. ${ }^{6}$ showed that increases in fat mass explained the increases in CRP levels during pubertal growth of Finnish girls but not vice versa.

BF\% was the only adiposity measure that showed discriminatory power for all the biomarkers in girls, but it should also be noted that, albeit BMI has presented marginally inferior AUC than BF\% for the rest of the biomarkers (except for CRP in girls and leptin for both sexes), still showed discriminatory power for most of them, and these differences from $\mathrm{BF} \%$ were not statistically significant.

Concerning the predictive power of the measures of abdominal adiposity we observed different trends. In girls, WHtR presented the highest AUC for most of the biomarkers, while for boys WC was the measure that more often provided better discriminatory power. We choose to include and test WsHtR due to its novel character and potential usefulness for screening obesity and related health risk, ${ }^{37}$ but it only presented the higher AUC when compared with the other abdominal adiposity measures for $\mathrm{C} 3$, in girls.

Some authors ${ }^{38,39}$ argue that measures of abdominal adiposity might be more useful predictors of cardiometabolic risk in non-obese adolescents, once excess abdominal adiposity could be missed if using a whole body measure such as BMI. There may also exist differences between sexes as Cartier et al. ${ }^{40}$ highlighted, although in adults, that CRP concentrations seem to be influenced to a greater extent by visceral adiposity in men, as 
opposed to subcutaneous adiposity in women.

In a study with healthy Spanish adolescents, ${ }^{41}$ the authors showed that although BMI and WC are strongly correlated and that both measures were good predictors of fat content in children and adolescents, they also report that different biomarkers associated with different adiposity measures. In that study C3, unlike CRP, C4 and ceruloplasmin, was preferentially associated with WC than BMI in both sexes after further adjustments for age, and pubertal maturity.

In another study, composed exclusively by German male adolescents, ${ }^{42} \mathrm{WHtR}$ presented a higher AUC for CRP than WC and WHR. In our study, WC was the measure of central adiposity that provided better discriminatory power for detecting CRP in boys. We observed similar results to Jung et al. ${ }^{42}$ only in girls.

According to Taylor et al., ${ }^{39}$ youths have the WC equivalent of adult abdominal obesity at percentiles lower than the frequently used pediatric threshold of $90 \%$. Besides, the WC cut-off values for pediatric ages have to be age and sex specific, so, a fixed cut-off point for WC not taking account of height might underestimate the relative amount of abdominal fat in short subjects and overestimate it in tall subjects. Likewise, some authors ${ }^{43-45}$ adjusted the WC to the person's height, proposing the WHtR as a way of assessing shape and monitoring risk reduction, first in adults, suggesting that values greater than 0.5 were indicative of increased health risks for both sexes, and showing then to be also valid to screen youth as young as five years old, ${ }^{46-48}$ making it interestingly age and sex independent. Systematic reviews and meta-analysis ${ }^{49-51}$ have been suggesting some superiority of WHtR over WC and BMI for detecting cardiometabolic risk factors in adults and children of both sexes, of various nationalities and ethnic groups.

In our study, although boys had higher values of WC it probably does not reflect larger intra-abdominal fat depots, because when the height of the subjects is considered, we observe that they are also significantly taller than girls, in a proportion that seems to correct this apparently abdominal obesity in boys. Indeed, there were no differences between sexes in the mean values of $\mathrm{WHtR}$ and WsHtR. In addition, the mean values of WC of the participants of both sexes are placed between the $50^{\text {th }}$ and the $75^{\text {th }}$ percentiles of Portuguese adolescents reference data. ${ }^{52}$ 
The cut-off points range for WHtR suggested by the ROC analysis in our study to predict increased levels of inflammatory markers in girls (0.44-0.48) and in boys (0.48-0.49) are slightly below the international proposed cut-off value of $\geq 0.5$ that is frequently associated with greater risks of overweight and metabolic syndrome, suggesting that even slightly below this threshold, some adolescents could present increased levels of some biomarkers. These are in line with other studies ${ }^{53,54}$ suggesting that a WHtR below 0.5 could predict adverse outcomes in youths. Nevertheless, further research focusing this thematic on pediatric ages is needed to confirm or contrast our findings.

The present study is not without limitations. First, its cross-sectional design does not allow us to assess the directionality of the relationships between the different adiposity measures and the different biomarkers; second, the use of a single measure of each biomarker may not accurately reflect a long-term inflammatory pattern of that specific biomarker; last, our sample is not nationally representative, and therefore these results cannot be extended to the entire population of Portuguese adolescents.

The strengths of this study include the analysis of various biomarkers, which provided us with a more accurate assessment of the inflammatory status of the adolescents, since we rely not only on a single marker; the utilization of various, simple, inexpensive and widespread measures of adiposity; the associations between those measures and several biomarkers should be further explored in the future, as they could be seen as indirect indicators of the inflammation profile of a sample of adolescents, particularly in epidemiological studies, when a large number of individuals are assessed and when blood samples may not be taken.

In conclusion, our results highlight the ability and utility of several anthropometric measures of overall and abdominal adiposity in detecting increased levels of biomarkers in adolescents. The measures that more often presented discriminatory power were, for overall adiposity, BF\% for both sexes, and in relation to abdominal adiposity, WHtR in girls and WC in boys. However, the small differences in the discriminatory capabilities between adiposity measures are perhaps of limited relevance, and based on our data it is difficult to clearly defend the adoption of a single measure of obesity in preference to all others. 


\section{ACKNOWLEDGEMENTS}

The Portuguese Foundation for Science and Technology (SFRH/BD/88984/2012; BPD/102381/2014; PTDC/DES/116586/2010; UID/DTP/00617/2013), the Research Centre in Physical Activity, Health and Leisure, University of Porto, the Coordination of Improvement of Higher Education Personnel (CAPES) from the Brazilian Government (Process: 9588-13-2), and the Australian Research Council (Discovery Early Career Research Award number DE150101921) supported this study.

\section{AUTHOR DISCLOSURE STATEMENT}

No competing financial interests exist. 


\section{REFERENCES}

1. Visser M. Higher levels of inflammation in obese children. Nutrition 2001;17:480-481.

2. Visser M, Bouter LM, McQuillan GM, et al. Low-grade systemic inflammation in overweight children. Pediatrics 2001;107:E13-E13.

3. Ford ES, Galuska DA, Gillespie C, et al. C-reactive protein and body mass index in children: findings from the Third National Health and Nutrition Examination Survey, 19881994. J Pediatr 2001;138:486-492.

4. Weiss R, Dziura J, Burgert TS, et al. Obesity and the Metabolic Syndrome in Children and Adolescents. N Engl J Med 2004;350:2362-2374.

5. Juonala M, Viikari JS, Ronnemaa T, et al. Childhood C-reactive protein in predicting CRP and carotid intima-media thickness in adulthood: the Cardiovascular Risk in Young Finns Study. Arterioscler Thromb Vasc Biol 2006;26:1883-1888.

6. Wen X, Pekkala S, Wang R, et al. Does systemic low-grade inflammation associate with fat accumulation and distribution? A 7-year follow-up study with peripubertal girls. J Clin Endocrinol Metab 2014;99:1411-1419.

7. Juonala M, Magnussen CG, Berenson GS, et al. Childhood adiposity, adult adiposity, and cardiovascular risk factors. N Engl J Med 2011;365:1876-1885.

8. Li C, Ford ES, Mokdad AH, et al. Recent trends in waist circumference and waistheight ratio among US children and adolescents. Pediatrics 2006;118:e1390-1398.

9. Cole TJ, Bellizzi MC, Flegal KM, et al. Establishing a standard definition for child overweight and obesity worldwide: international survey. BMJ: British Medical Journal 2000;320:1240-1243.

10. WHO. Obesity, preventing and managing the global epidemic: report of a WHO consultation Singapore2000 [cited 201515 January 2015]. Available from: http://whqlibdoc.who.int/trs/WHO_TRS_894.pdf. Last accessed July 3, 2015.

11. Berg AH, Scherer PE. Adipose tissue, inflammation, and cardiovascular disease. Circ Res 2005;96:939-949.

12. Freedman DS, Serdula MK, Srinivasan SR, et al. Relation of circumferences and skinfold thicknesses to lipid and insulin concentrations in children and adolescents: the Bogalusa Heart Study. Am J Clin Nutr 1999;69:308-317.

13. Steene-Johannessen J, Kolle E, Reseland JE, et al. Waist circumference is related to low-grade inflammation in youth. Int J Pediat Obes 2010;5:313-319.

14. El-Wakkad A, Hassan Nel M, Sibaii $\mathrm{H}$, et al. Proinflammatory, anti-inflammatory cytokines and adiponkines in students with central obesity. Cytokine 2013;61:682-687.

15. Khoury $\mathrm{M}$, Manlhiot $\mathrm{C}$, McCrindle BW. Role of the waist/height ratio in the cardiometabolic risk assessment of children classified by body mass index. J Am Coll Cardio 2013;62:742-751.

16. Pou KM, Massaro JM, Hoffmann U, et al. Visceral and subcutaneous adipose tissue volumes are cross-sectionally related to markers of inflammation and oxidative stress: the Framingham Heart Study. Circulation 2007;116:1234-1241.

17. Huh J, Riggs NR, Spruijt-Metz D, et al. Identifying patterns of eating and physical activity in children: a latent class analysis of obesity risk. Obesity 2011;19:652-658.

18. Ridker PM. Clinical application of C-reactive protein for cardiovascular disease detection and prevention. Circulation 2003;107:363-369.

19. Lohman T, Roche A, Martorell R. Human Kinetics Book Cl, editor. Anthropometric Standardization Reference Manual. Ilinois. 1988. 184 p.

20. Cole TJ, Flegal KM, Nicholls D, et al. Body mass index cut offs to define thinness in children and adolescents: international survey. BMJ 2007;335:194.

21. Talma H, Chinapaw MJ, Bakker B, et al. Bioelectrical impedance analysis to estimate body composition in children and adolescents: a systematic review and evidence appraisal of validity, responsiveness, reliability and measurement error. Obes Rev 2013;14:895-905.

22. Abreu S, Santos R, Moreira C, et al. Relationship of milk intake and physical activity to abdominal obesity among adolescents. Pediatr Obes 2014;9:71-80.

23. Tanner JM. Blackwell, editor. Growth at adolescence. Oxford. Blackwell Scientific Publications; 1962. $326 \mathrm{p}$. 
24. Cohen JI, Maayan L, Convit A. Preliminary evidence for obesity-associated insulin resistance in adolescents without elevations of inflammatory cytokines. Diabetol Metab Syndr 2012;4:26.

25. Guran O, Akalin F, Ayabakan C, et al. High-sensitivity C-reactive protein in children at risk for coronary artery disease. Acta Paediatr 2007;96:1214-1219.

26. Labayen I, Ortega FB, Sjostrom M, et al. Early life origins of low-grade inflammation and atherosclerosis risk in children and adolescents. J Pediatr 2009;155:673-677.

27. Martinez-Gomez D, Eisenmann JC, Gomez-Martinez S, et al. Sleep duration and emerging cardiometabolic risk markers in adolescents. The AFINOS study. Sleep Med 2011;12:997-1002.

28. Brooks GC, Blaha MJ, Blumenthal RS. Relation of C-reactive protein to abdominal adiposity. Am J Cardiol 2010;106:56-61.

29. Vikram NK, Misra A, Dwivedi M, et al. Correlations of C-reactive protein levels with anthropometric profile, percentage of body fat and lipids in healthy adolescents and young adults in urban North India. Atherosclerosis 2003;168:305-313.

30. Blum WF, Englaro $\mathrm{P}$, Hanitsch S, et al. Plasma leptin levels in healthy children and adolescents: dependence on body mass index, body fat mass, gender, pubertal stage, and testosterone. J Clin Endocrinol Metab 1997;82:2904-2910.

31. Martinez-Gomez D, Eisenmann JC, Gomez-Martinez S, et al. Associations of physical activity and fitness with adipocytokines in adolescents: the AFINOS Study. Nutr Metab Cardiovasc Dis 2012;22:252-259.

32. Tsou PL, Jiang YD, Chang CC, et al. Sex-related differences between adiponectin and insulin resistance in schoolchildren. Diabetes Care 2004;27:308-313.

33. Ford ES. C-reactive protein concentration and cardiovascular disease risk factors in children: findings from the National Health and Nutrition Examination Survey 1999-2000. Circulation 2003;108:1053-1058.

34. Ford ES, Ajani UA, Mokdad AH. The Metabolic Syndrome and Concentrations of CReactive Protein Among U.S. Youth. Diabetes Care 2005;28:878-881.

35. Choi J, Joseph L, Pilote L. Obesity and C-reactive protein in various populations: a systematic review and meta-analysis. Obes Rev 2013;14:232-244.

36. Freedman DS, Wang J, Maynard LM, et al. Relation of BMI to fat and fat-free mass among children and adolescents. Int J Obes 2005;29:1-8.

37. Zhang $\mathrm{YX}$, Zhao JS, Chu ZH. Waist to sitting height ratio may be a new useful index for screening obesity and related health risk. Int J Cardiol 2015;187:126-127.

38. Lee S, Bacha F, Gungor N, et al. Waist circumference is an independent predictor of insulin resistance in black and white youths. J Pediatr 2006;148:188-194.

39. Taylor SA, Hergenroeder AC. Waist circumference predicts increased cardiometabolic risk in normal weight adolescent males. Int J Pediatr Obes 2011;6:e307-311.

40. Cartier A, Côté M, Lemieux I, et al. Sex differences in inflammatory markers: what is the contribution of visceral adiposity? Am J Clin Nutr 2009;89:1307-1314.

41. Warnberg J, Nova E, Moreno LA, et al. Inflammatory proteins are related to total and abdominal adiposity in a healthy adolescent population: the AVENA Study. Am J Clin Nutr 2006;84:505-512.

42. Jung $\mathrm{C}$, Fischer $\mathrm{N}$, Fritzenwanger $\mathrm{M}$, et al. Anthropometric indices as predictors of the metabolic syndrome and its components in adolescents. Pediatr Int 2010;52:402-409.

43. Ashwell M, Cole TJ, Dixon AK. Ratio of waist circumference to height is strong predictor of intra-abdominal fat. BMJ 1996;313:559-560.

44. Cox BD, Whichelow M. Ratio of waist circumference to height is better predictor of death than body mass index. BMJ : British Medical Journal 1996;313:1487-1487.

45. Hsieh SD, Yoshinaga $\mathrm{H}$. Abdominal fat distribution and coronary heart disease risk factors in men-waist/height ratio as a simple and useful predictor. Int J Obes Relat Metab Disord 1995;19:585-589.

46. Garnett SP, Baur LA, Cowell CT. Waist-to-height ratio: a simple option for determining excess central adiposity in young people. Int J Obes 2008;32:1028-1030.

47. McCarthy HD, Ashwell M. A study of central fatness using waist-to-height ratios in UK children and adolescents over two decades supports the simple message--'keep your waist circumference to less than half your height'. Int J Obes 2006;30:988-992.

48. Nambiar S, Truby H, Abbott RA, et al. Validating the waist-height ratio and developing centiles for use amongst children and adolescents. Acta Paediatr 2009;98:148-152. 
49. Ashwell M, Gunn P, Gibson S. Waist-to-height ratio is a better screening tool than waist circumference and BMI for adult cardiometabolic risk factors: systematic review and meta-analysis. Obes Rev 2012;13:275-286.

50. Browning LM, Hsieh SD, Ashwell M. A systematic review of waist-to-height ratio as a screening tool for the prediction of cardiovascular disease and diabetes: 0.5 could be a suitable global boundary value. Nutr Res Rev 2010;23:247-269.

51. Lee CM, Huxley RR, Wildman RP, et al. Indices of abdominal obesity are better discriminators of cardiovascular risk factors than BMI: a meta-analysis. J Clin Epidemiol 2008;61:646-653.

52. Sardinha LB, Santos R, Vale S, et al. Waist circumference percentiles for Portuguese children and adolescents aged 10 to 18 years. Eur J Pediatr 2012;171:499-505.

53. Graves L, Garnett SP, Cowell CT, et al. Waist-to-height ratio and cardiometabolic risk factors in adolescence: findings from a prospective birth cohort. Pediatr Obes 2014;9:327338.

54. Nambiar S, Hughes I, Davies PS. Developing waist-to-height ratio cut-offs to define overweight and obesity in children and adolescents. Public Health Nutr 2010;13:1566-1574. 
Table 1. Anthropometric, biochemical and maturational characteristics of the participants.

\begin{tabular}{|c|c|c|c|}
\hline & All $(n=529)$ & Girls $(n=267)$ & Boys $(n=262)$ \\
\hline Variables & Mean \pm SD & Mean \pm SD & Mean \pm SD \\
\hline Age (years) & $14.3 \pm 1.7$ & $14.3 \pm 1.7$ & $14.4 \pm 1.7$ \\
\hline Height (cm) & $160.3 \pm 9.6$ & $157.7 \pm 6.7$ & $162.9 \pm 11.3$ * \\
\hline Weight (kg) & $55.2 \pm 12.8$ & $53.4 \pm 11.2$ & $56.9 \pm 14.1$ * \\
\hline BMI $\left(\mathrm{kg} / \mathrm{m}^{2}\right)$ IOTF & $21.31 \pm 3.84$ & $21.41 \pm 3.96$ & $21.20 \pm 3.73$ \\
\hline UW/ NW/ OW/ OB (n) & $24 / 357 / 111 / 37$ & $10 / 181 / 54 / 22$ & $14 / 176 / 57 / 15$ \\
\hline UW/ NW/ OW/ OB (\%) & $4.5 \% / 67.5 \% / 21 \% / 7 \%$ & $3.7 \% / 67.8 \% / 20.3 \% / 8.2 \%$ & $5.3 \% / 67.2 \% / 21.8 \% / 5.7 \%$ \\
\hline $\mathrm{BF} \%$ & $20.7 \pm 8.3$ & $25.4 \pm 7.0$ & $15.9 \pm 6.7$ * \\
\hline WC (cm) & $73.1 \pm 10.2$ & $72 \pm 10.2$ & $74.2 \pm 10.2$ * \\
\hline WHtR & $0.45 \pm 0.06$ & $0.45 \pm 0.6$ & $0.46 \pm 0.6$ \\
\hline WsHtR & $0.86 \pm 0.11$ & $0.86 \pm 0.12$ & $0.87 \pm 0.1$ \\
\hline CRP (mg/L) & $0.95 \pm 1.88$ & $0.77 \pm 1.59$ & $1.13 \pm 2.12$ * \\
\hline Fibrinogen (mg/dL) & $264 \pm 43.2$ & $268.13 \pm 41.72$ & $259.77 \pm 44.43$ * \\
\hline Adiponectin (mg/L) & $11.61 \pm 5.45$ & $12.90 \pm 5.74$ & $10.29 \pm 4.79$ * \\
\hline Complement C3 (g/L) & $1.17 \pm 0.16$ & $1.18 \pm 0.16$ & $1.17 \pm 0.16$ \\
\hline Complement C4 (g/L) & $0.21 \pm 0.06$ & $0.21 \pm 0.06$ & $0.21 \pm 0.06$ \\
\hline $\mathrm{ESR}(\mathrm{mm} / \mathrm{h})$ & $6.22 \pm 6.15$ & $7.44 \pm 6.47$ & $4.98 \pm 5.53$ * \\
\hline \multirow[t]{2}{*}{ Leptin $(\mathrm{ng} / \mathrm{mL})$} & $4.12 \pm 4.93$ & $6.21 \pm 5.6$ & $1.98 \pm 2.85$ * \\
\hline & n (\%) & $n(\%)$ & n (\%) \\
\hline \multicolumn{4}{|l|}{ Tanner A } \\
\hline Stage $\leq 2$ & $41(7.8)$ & $8(3)$ & $33(12.6)$ \\
\hline Stage 3 & $171(32.3)$ & $78(29.2)$ & $93(35.5)$ \\
\hline Stage 4 & $247(46.7)$ & $145(54.3)$ & $102(38.9)$ \\
\hline Stage 5 & $70(13.2)$ & $36(13.5)$ & $34(13.0)$ \\
\hline \multicolumn{4}{|l|}{ Tanner B } \\
\hline Stage $\leq 2$ & $37(7)$ & $6(2.2)$ & $31(11.8)$ \\
\hline Stage 3 & $115(21.7)$ & $64(24)$ & 51 (19.5) \\
\hline Stage 4 & $262(49.5)$ & $125(46.8)$ & $137(52.3)$ \\
\hline Stage 5 & $115(21.8)$ & $72(27)$ & $43(16.4)$ \\
\hline
\end{tabular}

$p<0.05$ for sex comparisons (two-tailed t-test).

$\mathrm{BF} \%$, body fat percentage; BMI, body mass index (according to the age and sex-specific cut-off values of the International Obesity Task Force [IOTF]); CRP, high sensivity C-reactive protein; ESR, erythrocyte sedimentation rate; NW, normal weight; OB, obese; OW, overweight;

SD, standard deviation; Tanner A, breast development in girls, genital development in boys; Tanner B, pubic hair development; UW, underweight; WC, waist circumference; WHtR, waist-to-height ratio; WsHtR, waist-to-sitting-height ratio. 
Table 2. AUC, $95 \% \mathrm{Cl}, p$ values, sensitivity, specificity and cut-off values for the associations of measures of overall and abdominal adiposity with inflammatory and metabolic markers for girls.

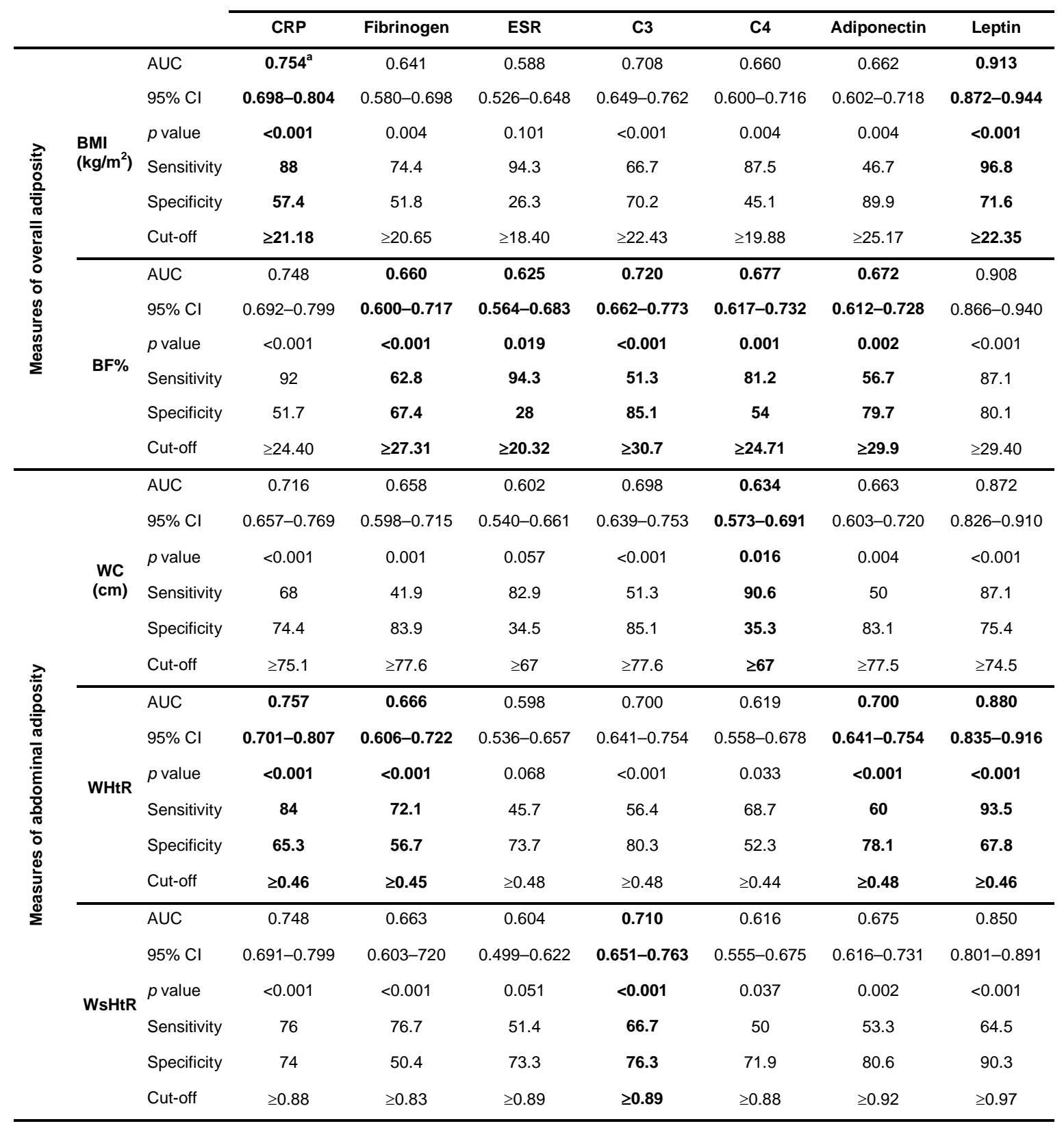

AUC, area under the curve; $\mathrm{Cl}$, confidence interval, BF\%, body fat percentage; BMI, body mass index; CRP, C-reactive protein; ESR, erythrocyte sedimentation rate; WC, waist circumference; WHtR, waist-to-height ratio; WsHtR, waist-to-sitting-height ratio.

${ }^{a}$ Values in bold represent the ROC curves indicating the adiposity measures that presented the higher AUC for each biomarker. 
Table 3. AUC, 95\% Cl, $p$ values, sensitivity, specificity and cut-off values for the associations of measures of overall and abdominal adiposity with inflammatory and metabolic markers for boys.

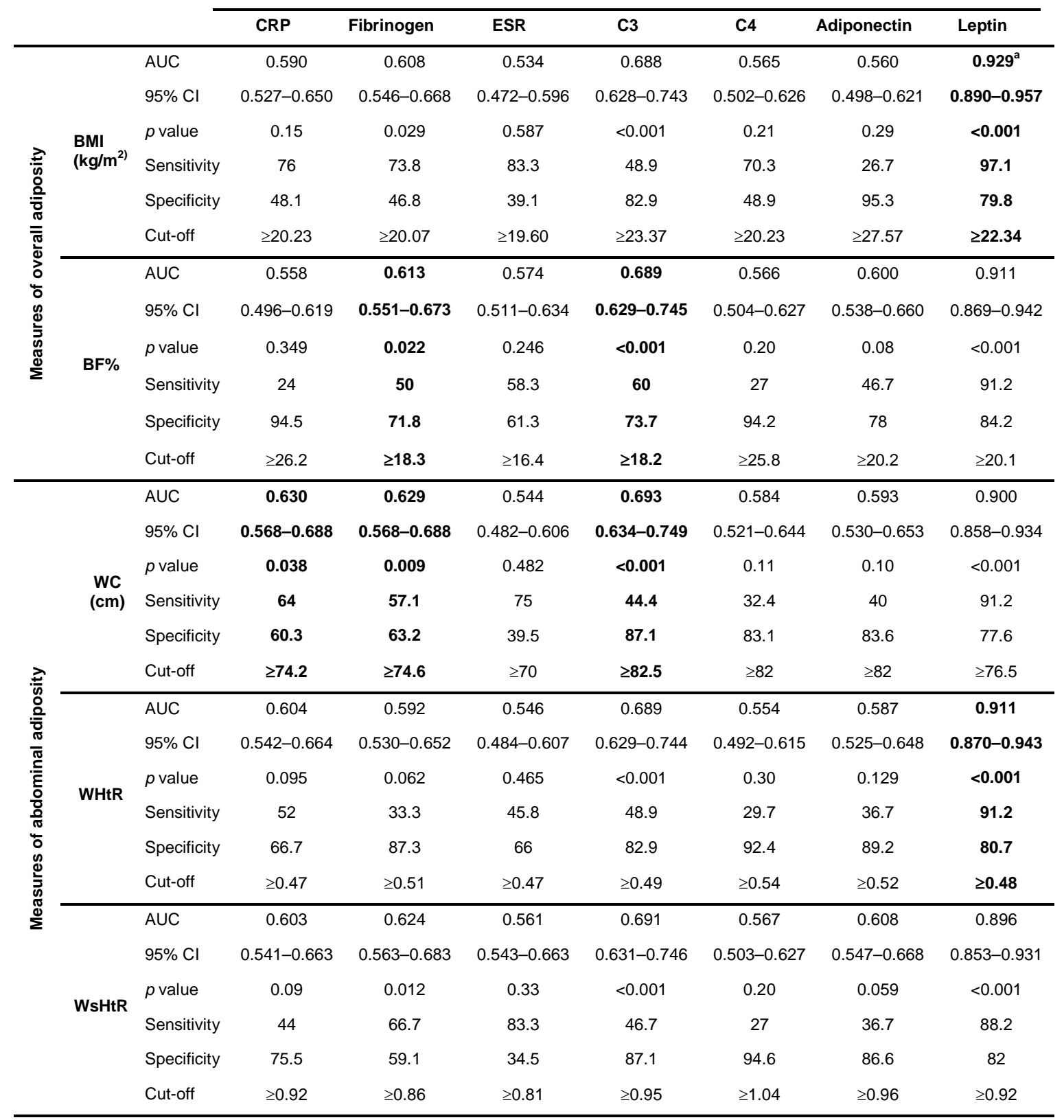

AUC, area under the curve; $\mathrm{Cl}$, confidence interval, BF\%, body fat percentage; BMI, body mass index; CRP, C-reactive protein; ESR, erythrocyte sedimentation rate; WC, waist circumference; WHtR, waist-to-height ratio; WsHtR, waist-to-sitting-height ratio.

${ }^{a}$ Values in bold represent the ROC curves indicating the adiposity measures that presented the higher AUC for each biomarker. 